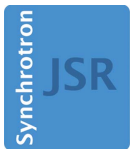

JOURNAL OF SYNCHROTRON RADIATION

ISSN 1600-5775

Received 10 July 2017

Accepted 24 October 2017

Edited by D. Cocco, SLAC National Accelerator Laboratory, USA

Keywords: FLASH; free-electron laser; $\mathrm{THz}$ beamline; pump-probe experiments; double electron bunches; X-rays.

Supporting information: this article has supporting information at journals.iucr.org/s

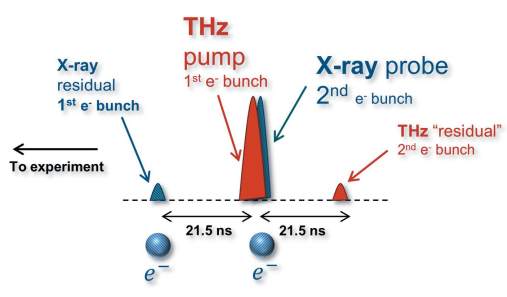

OPEN $\odot$ ACCESS

\section{THz pulse doubler at FLASH: double pulses for pump-probe experiments at X-ray FELs}

\author{
Ekaterina Zapolnova, Torsten Golz, Rui Pan, Karsten Klose, Siegfried Schreiber and \\ Nikola Stojanovic*
}

Deutsches Elektronen-Synchrotron - DESY, Notkestrasse 85, D-22607 Hamburg, Germany

*Correspondence e-mail: nikola.stojanovic@desy.de

FLASH, the X-ray free-electron laser in Hamburg, Germany, employs a narrowband high-field accelerator $\mathrm{THz}$ source for unique $\mathrm{THz}$ pump X-ray probe experiments. However, the large difference in optical paths of the $\mathrm{THz}$ and X-ray beamlines prevents utilization of the machine's full potential (e.g. extreme pulse energies in the soft X-ray range). To solve this issue, lasing of double electron bunches, separated by 28 periods of the driving radiofrequency (at $1.3 \mathrm{GHz}$ ), timed for the temporal overlap of $\mathrm{THz}$ and $\mathrm{X}$-ray pulses at the experimental station has been employed. In order to optimize conditions for a typical THz pump X-ray probe experiment, X-ray lasing of the first bunch to one-sixth of that of the second has been suppressed. Finally, synchronization of $\mathrm{THz}$ radiation pulses was measured to be $\sim 20 \mathrm{fs}$ (r.m.s.), and a solution for monitoring the arrival time for achieving higher temporal resolution is presented.

\section{Introduction}

The X-ray free-electron laser FLASH1 at DESY in Hamburg, Germany, has a worldwide unique feature - a THz undulator behind the main X-ray undulator section, that allows users to perform pump-probe experiments with ultrashort highintensity $\mathrm{THz}$ and soft X-ray pulses (Gensch et al., 2008; Frühling et al., 2009; Tavella et al., 2011; Stojanovic \& Drescher, 2013; Oelze et al., 2017). The wavelength range can be tuned from 1 to $300 \mu \mathrm{m}$ ( 1 to $300 \mathrm{THz}$ ), depending on the electron beam energy. $\mathrm{THz}$ and $\mathrm{X}$-ray pulses are produced by the same electron bunch, and are naturally synchronized (down to 5 fs) (Frühling et al., 2009; Tavella et al., 2011). However, a problem that plagues this type of experiment is that $\mathrm{THz}$ and $\mathrm{X}$-ray optical paths are significantly different. In the beamline, the $\mathrm{THz}$ beam is collimated using five toroidal mirrors to keep the beam size within the size of the beam transport and optics (size $210 \mathrm{~mm} \times 150 \mathrm{~mm}$ ). Due to the extremely broad spectral range that the undulator covers, all optical elements are reflective (providing spectrally nondispersive reflection) and provide a $45^{\circ}$ angle of incidence. Folding of the $\mathrm{THz}$ beam adds additional optical path, adding up to a delay of $21.5 \mathrm{~ns}(\sim 6.5 \mathrm{~m}$ of optical path) with respect to X-ray pulses at the experimental station. One solution to ensure temporal overlap between $\mathrm{THz}$ and $\mathrm{X}$-ray pulses at the experiment is to introduce additional optical path for the X-rays in back-reflection geometry (Feldhaus et al., 2005; Frühling et al., 2009; Oelze et al., 2017) using normal-incidence multilayer mirrors specially designed for a particular wavelength (Bajt et al., 2007). Although this method has been successfully demonstrated (Frühling et al., 2009; Oelze et al., 
2017), there are two major drawbacks when utilizing this technique (within the specificities of the discussed setup): low reflectivity and narrow spectral range of the X-ray mirrors. Although not a limitation of the multilayer mirror design, this geometry also suffers from a large focal size of the X-ray beam ( $>100 \mu \mathrm{m}$ FWHM), caused by the large focal length $(3.5 \mathrm{~m})$ determined by the difference in optical path between $\mathrm{THz}$ and $\mathrm{X}$-ray pulses. Reduction of the X-ray fluence in the experiment due to the combined effect of low reflectivity and large focus excludes high-intensity experiments [e.g. plasma physics (Nagler et al., 2009) or non-linear multiphoton ionizations (Richter et al., 2009, 2010)] thus limiting FLASH's high pulse energy advantage (Rönsch-Schulenburg et al., 2017) over other sources in the same spectral range. An alternative approach which avoids the need of introducing a delay for the X-ray pulses, proposed by Grimm et al. (2006), is to exploit the radiation of tailored electron bunches. In particular, we generate double electron bunches at the electron gun, separated by $21.5 \mathrm{~ns}$. In this scheme the first electron bunch is used to generate $\mathrm{THz}$ and the second to generate soft X-ray pulses. Each double electron bunch is generated by two consecutive laser pulses illuminating the photocathode at FLASH's electron gun.

For typical $\mathrm{THz}$ pump/X-ray probe experiments the first electron bunch needs to be optimized to generate maximal THz pulse energy (refer to scheme in Fig. 1), while generating little or no X-rays, to reduce their potential influence on the sample. The second bunch, on the other hand, should be optimized to generate X-ray pulses with required properties, ranging from short pulse duration to high pulse energy, while suppressing the $\mathrm{THz}$ output (although this is less critical).

In this work we present the generation of $\mathrm{THz}$ pulses from double electron bunches, studying the energy-ratio control of individual $\mathrm{THz}$ and X-ray pulses and, most importantly, the arrival-time stability of two $\mathrm{THz}$ doubler pulses essential for accurate pump-probe experiments. By doing this we are opening the pathway to utilizing the full potential of FLASH's high pulse energies in the synchronized THz/X-ray pumpprobe experiments.

\section{Concept of double-bunch generation}

The concept of the double pulse generation at FLASH $(\mathrm{THz}$ doubler) has been demonstrated elsewhere (Grimm et al., 2006) and the main issues for transport through the accelerator have been identified. Here, we briefly refer to it for completeness (refer to Fig. 2).

The fourth harmonic of the driving photocathode laser, at $262 \mathrm{~nm}$, is split by a polarization beamsplitter (fused silica at the Brewster angle). One of the branches is delayed by $21.5 \mathrm{~ns}$ with the option to fine-tune the arrival time of the second pulse via a delay stage (e.g. for adjusting it to the phases of the accelerating modules). Divergence and size resulting from the large difference in optical path are compensated for by a Keplerian telescope. The two beams are recombined by a polarization recombiner and brought together at a photo-

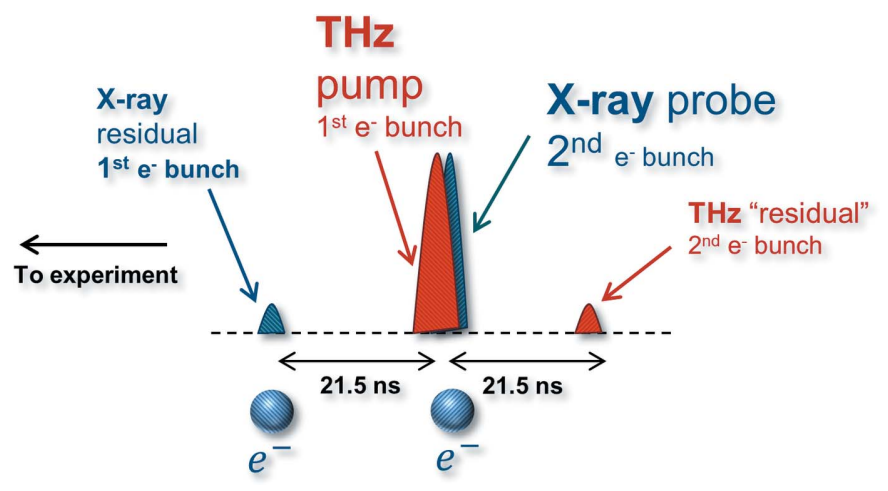

Figure 1

$\mathrm{THz}$ doubler. Schematic representation of the X-ray and $\mathrm{THz}$ pulse arrival times for the double electron bunch scheme.

cathode. Each of the pulses can be blocked by closing the corresponding shutter.

\section{Characterization of $\mathrm{THz}$ doubler radiation}

Double electron bunches for the $\mathrm{THz}$ doubler have been set up following the procedure described by Grimm et al. (2006). Electron bunch charges were almost equal and their arrival time with respect to the accelerating radiofrequency $(\mathrm{RF})$ was adjusted to facilitate electron beam transmission through the accelerator.

In the following we present the characterization of $\mathrm{THz}$ and $\mathrm{X}$-ray pulse energies and a novel technique to determine the relative arrival time of individual $\mathrm{THz}$ pulses. The X-ray pulse arrival time for the same electron bunch is locked to the respective $\mathrm{THz}$ pulse down to a few femtoseconds due to natural synchronization (Frühling et al., 2009; Tavella et al., 2011; Oelze et al., 2017).

\subsection{THz pulse energy measurements}

The THz undulator at FLASH was set to $140 \mu \mathrm{m}$ wavelength and the individual electron bunches had charges of $0.3 \mathrm{nC}$ (direct, first) and $0.25 \mathrm{nC}$ (delayed, second). We

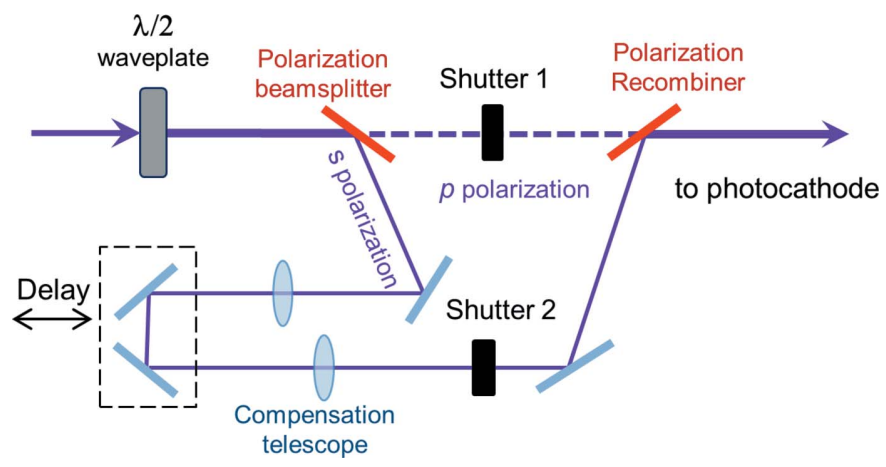

Figure 2

Scheme of the laser pulse doubler [adapted from Grimm et al. (2006)]. The laser pulse is split, one pulse is delayed and then recombined with a direct one. Each branch includes a shutter, so that each pulse can be selected at will. 
measured $\mathrm{THz}$ pulse energies using a radiometer [RM3700, head RjP-735/RF, by Laser Probe, cross-referenced to PTB traceable 3A-P-THz radiometer (Green et al., 2016)]. The detector had a cavity pyroelectric probe and its time constant of $1 \mathrm{~ms}$ prevented us from resolving individual pulses of the $\mathrm{THz}$ doubler. Thus, pulse energies were measured as an average over a few hundred pulses. Selection of individual $\mathrm{THz}$ doubler pulses was achieved by closing the corresponding shutter in the injector setup. The first pulse (shutter 1 open, shutter 2 closed) measured $1.05 \pm 0.07 \mu \mathrm{J}$, the second pulse (shutter 1 closed, shutter 2 open) measured $0.84 \pm 0.06 \mu \mathrm{J}$, and both pulses together measured $1.94 \pm 0.13 \mu \mathrm{J}$. Hereby we confirm that electron bunches with similar charge and experiencing the same accelerator conditions (e.g. compression) yield similar $\mathrm{THz}$ pulse energies.

In the future, we plan to explore adjusting the relative pulse energies in the $\mathrm{THz}$ doubler and the influence on the SASE levels. One possibility is to adjust the ratio of the pulse energies illuminating the photocathode by adjusting the polarization of the laser pulse impinging on the beamsplitter in the laser doubler setup. The second option is by adjusting the arrival time of the second pulse with respect to the RF phase in the accelerating module, utilizing the optical delay stage in the respective branch of the photocathode laser.

\subsection{SASE pulse energy measurements}

$\mathrm{X}$-ray pulse energies are measured using one of the gas monitor detectors installed at FLASH1 (Tiedtke et al., 2008). Temporal resolution of these detectors is in the region of $100 \mathrm{~ns}$. Thus individual X-ray pulses generated by the $\mathrm{THz}$ doubler cannot be resolved. The average ratio of the X-ray pulse energy is retrieved by successively measuring the first and second pulse, as for the $\mathrm{THz}$ pulses before. The pulse energy of one bunch was measured by blocking the other branch in the electron gun laser. Fig. 3 displays pulse energy measurements for the first and second pulses. Initially, the first electron bunch, with $0.3 \mathrm{nC}$ charge, produced $40 \pm 6 \mu \mathrm{J}$, while

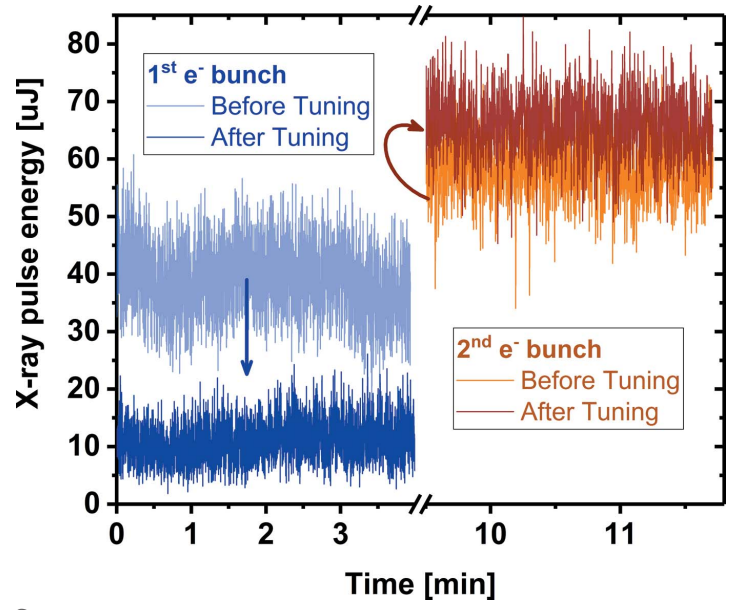

Figure 3

X-ray SASE pulse energies for the first and second pulse of the $\mathrm{THz}$ doubler. From initially similar levels, the pulse energy of the first bunch has been suppressed to one-sixth of the intensity of the second. the second, with $0.25 \mathrm{nC}$, produced $58 \pm 5 \mu \mathrm{J}$. After optimization by shifting the relative arrival time of each individual laser doubler pulse to the RF phase, the average energy of the first and the second pulses was $11 \pm 4 \mu \mathrm{J}$ and $67 \pm 6 \mu \mathrm{J}$, respectively. These results show the maximum achieved suppression of the first SASE resulting in a ratio of 1:6.

There are several ways to further suppress X-ray lasing of the first bunch. For example, by introducing a slight kick $(\sim 100 \mu \mathrm{m}$ in the vertical direction) in the electron bunch orbit in the X-ray undulators, it has been shown that the SASE process can be suppressed by two orders of magnitude (Först et al., 2011), while maintaining similar pulse energies in the $\mathrm{THz}$ spectral range (refer to $\S \mathrm{S} 3$ in the supporting information).

\subsection{Arrival-time stability}

Electro-optic spectral decoding (EOSD) detection (Jiang \& Zhang, 1998; Steffen, 2007) maps the THz field onto a temporally chirped probing laser pulse in a single shot. It can be used as an arrival-time marker of $\mathrm{THz}$ to the external laser (Kovalev et al., 2017). We adapt this technique to detect the arrival time of the $\mathrm{THz}$ doubler pulses simultaneously (refer to Fig. 4) using a single probe laser pulse. The laser pulse (30 fs FWHM at $860 \mathrm{~nm}$ ) is split by a non-polarizing beamsplitter and one pulse is delayed by $21.5 \mathrm{~ns}$. Pulses are then recombined, using the same type of non-polarizing beamsplitter, and follow the same optical path thereafter. Since the laser pulse has been split optically, temporal instabilities are minimal. Both laser pulses are stretched in a $450 \mathrm{~mm}$ glass (SF57) stretcher to linearly chirped pulses with $16.5 \mathrm{ps}$ FWHM duration. The stretched pulses are overlapped (spatially and temporally) with $\mathrm{THz}$ doubler $\mathrm{THz}$ pulses in an electro-optic (EO) crystal.

The electric field of the THz pulses induces birefringence in the EO crystal $(1 \mathrm{~mm} \mathrm{ZnTe})$ that modulates polarization of the probing laser pulses. Polarization modulation is transferred into a modulation of intensity by an analyser (Wollaston prism). Laser pulses are sent to a grating spectrometer (Princeton Instruments, Acton SP2300i) and detected using a line detector (Basler Sprint Mono spL-2048$70 \mathrm{~km}$ ). The detector response is slower than the time delay between the pulses, which is used to observe the traces of both pulses in the same spectral recording. In order to be able to detect the respective arrival time between the two $\mathrm{THz}$ doubler bunches, the arrival time of the probe laser pulses is adjusted so that the $\mathrm{THz}$ doubler pulses modulate different spectral regions of their respective probe laser pulses (refer to Fig. 5 and $\S S 1$ of the supporting information). The modulations are well separated to avoid ambiguities in arrival-time determination.

The THz pulses in this example are generated by FLASH's electron beam dump magnet by the edge radiation process (Geloni et al., 2009; Tavella et al., 2011; Stojanovic \& Drescher, 2013). The profile of single-cycle $\mathrm{THz}$ pulses measured by EOSD deviates from our previous results [e.g. by $\mathrm{THz}$ streaking (Stojanovic \& Drescher, 2013) or EO sampling 


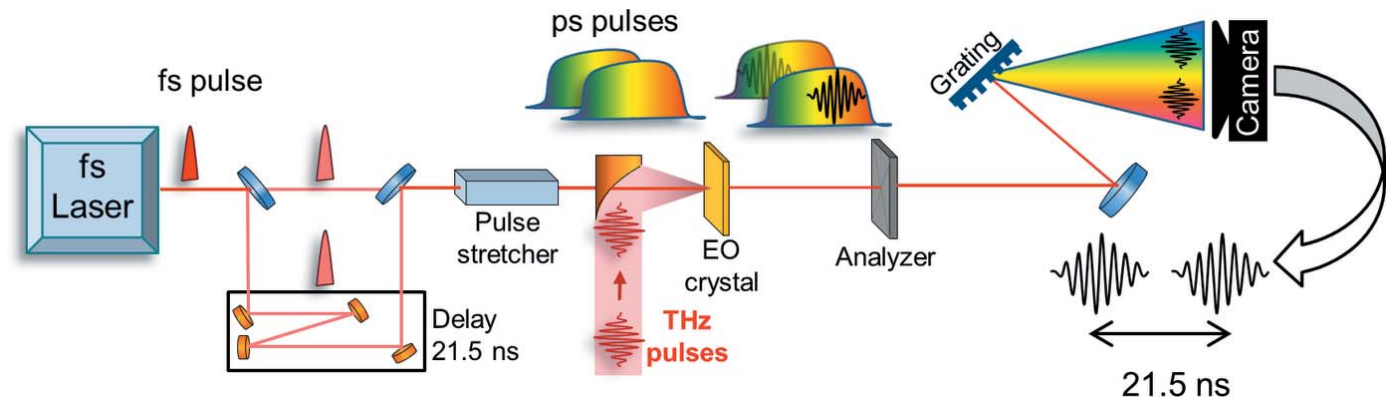

Principle of double $\mathrm{THz}$ pulses arrival-time measurement based on the spectral decoding technique.

(Golz et al., 2018)], mainly because of a characteristic of this technique that the frequency mixing between the short $\mathrm{THz}$ pulse and the chirped laser pulse leads to a detection distortion (Jamison et al., 2008; Pan, 2015). However, the measured distorted profiles keep the same shape within the timing jitter range, and therefore the arrival time of the pulses is not affected due to causality (also confirmed during temporal calibration of the EOSD; refer to $\S \mathrm{S} 2$ of the supporting information).

A typical series of subsequent shots is presented in Fig. 6(a). From each trace we extract the arrival time of each individual $\mathrm{THz}$ doubler pulse with respect to the corresponding probe laser pulse [black curve in Fig. 6(a)]. Their difference gives their relative temporal jitter [red curve in Fig. 6(a)].

The overall level of jitter measured over a period of $12 \mathrm{~min}$ is estimated to be 23 fs (r.m.s.). We observe that jitter between $\mathrm{THz}$ pulses and probing laser ( 96 fs r.m.s.) is much larger than that between $\mathrm{THz}$ doubler pulses. We also performed jitter measurements for the $\mathrm{THz}$ undulator set to $140 \mu \mathrm{m}$ wave-
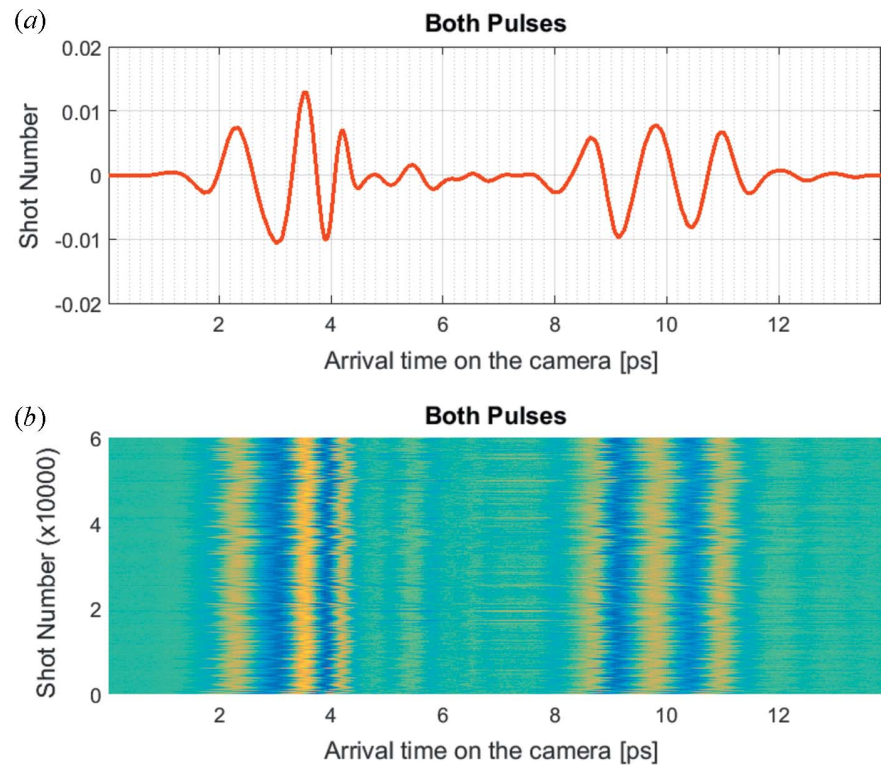

Figure 5

Spectral decoding detection of the THz doubler pulses generated by an electron beam dump magnet (edge radiation). (a) Single-shot trace, modulations with the first and the second $\mathrm{THz}$ pulse well separated. (b) Series of single-shot measurements acquired over $\sim 90 \mathrm{~min}(60000$ shots). length. The jitter between narrowband $\mathrm{THz}$ doubler pulses is estimated to be on the level of $18 \mathrm{fs}$ (r.m.s.) over a period of $15 \mathrm{~min}$.

\section{Conclusion}

We have demonstrated the feasibility of the $\mathrm{THz}$ doubler concept, accelerating and lasing of the double electron bunches timed for the temporal overlap of $\mathrm{THz}$ and $\mathrm{X}$-ray pulses in user experiments. In the case of FLASH, electron bunches are separated by 28 periods of the driving RF (at $1.3 \mathrm{GHz}$ ) or $21.5 \mathrm{~ns}$. We observed $\mathrm{THz}$ pulse energies in
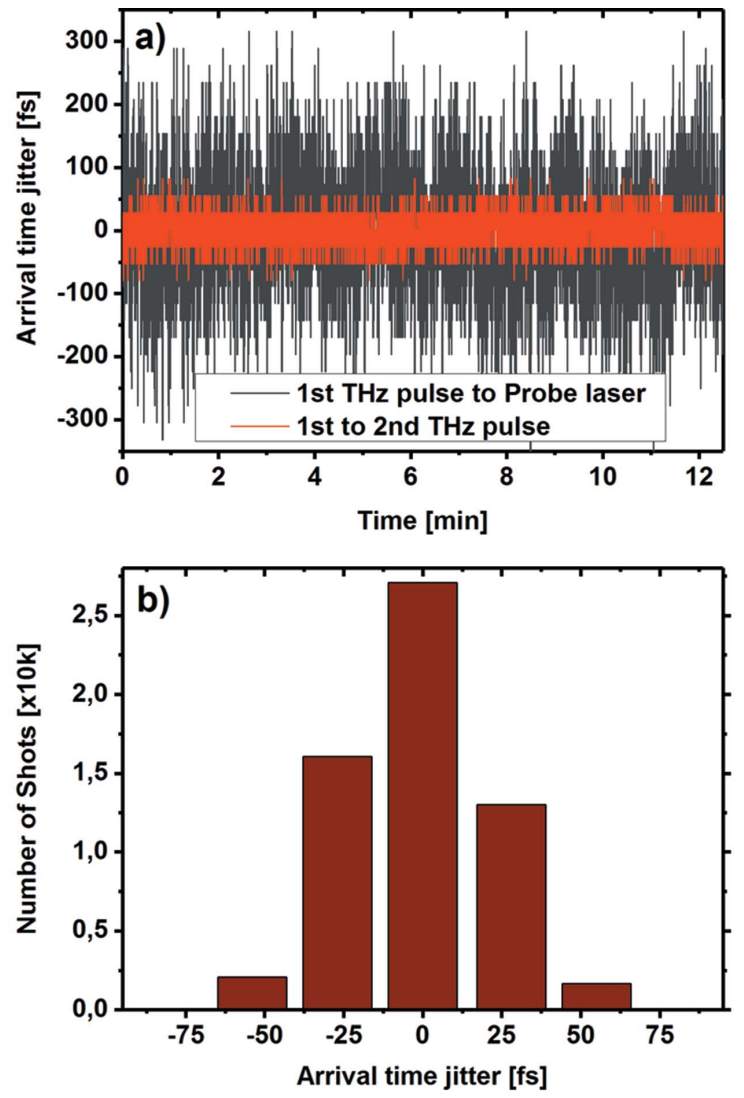

Figure 6

(a) Arrival-time jitter of the first $\mathrm{THz}$ pulse with respect to the laser (black), and jitter between the two THz pulses (red). (b) The distribution of the jitter between individual $\mathrm{THz}$ doubler pulses has a width of $23 \mathrm{fs}$ (r.m.s.). 
the microjoule range and, critically, we have demonstrated suppression of the X-ray pulse energy of the first electron bunch $(11 \mu \mathrm{J})$, down to a level of $16 \%$ of the second bunch $(67 \mu \mathrm{J})$ of the $\mathrm{THz}$ doubler. This will significantly widen the range of experiments that can utilize this setup. This suppression can be further improved (by two orders of magnitude) if fast kickers are employed to deflect the electron bunch orbit by $100 \mu \mathrm{m}$ in the X-ray undulators. Finally, we observed that the arrival-time stability of individual $\mathrm{THz}$ pulses in the $\mathrm{THz}$ doubler is around $20 \mathrm{fs}$ (r.m.s.). For experiments where a more accurate arrival time is required, spectral decoding presented in this work provides a photonefficient tool for monitoring the arrival time for data sorting (Azima et al., 2009; Kovalev et al., 2017). Since the tool requires only modest laser power (e.g. femtosecond oscillator), it can be readily applied at any similar facility (Svetina et al., 2016) or can be extended for use at larger-scale X-ray free-electron lasers like the European XFEL or LCLS II. The $\mathrm{THz}$ doubler has the potential to solve the issue that any of the $\mathrm{X}$-ray free-electron lasers with an integrated $\mathrm{THz}$ source will face: a large difference in the optical path between X-rays and accelerator-based $\mathrm{THz}$ pulses.

\section{Related literature}

The following reference, not cited in the main body of the paper, has been cited in the supporting information: Behrens et al. (2010).

\section{Acknowledgements}

The authors would like to acknowledge Bart Faatz, Wilfried Wurth and Jovana Petrovic for fruitful discussions.

\section{Funding information}

The following funding is acknowledged: Deutscher Akademischer Austauschdienst (grant No. 57219839 to NS); Bundesministerium für Bildung und Forschung (grant No. $05 \mathrm{~K} 12 \mathrm{CH} 4$ to NS).

\section{References}

Azima, A., Düsterer, S., Radcliffe, P., Redlin, H., Stojanovic, N., Li, W., Schlarb, H., Feldhaus, J., Cubaynes, D., Meyer, M., Dardis, J., Hayden, P., Hough, P., Richardson, V., Kennedy, E. T. \& Costello, J. T. (2009). Appl. Phys. Lett. 94, 144102.

Bajt, S., Chapman, H. N., Spiller, E., Hau-Riege, S., Alameda, J., Nelson, A. J., Walton, C. C., Kjornrattanawanich, B., Aquila, A., Dollar, F., Gullikson, E. \& Tarrio, C. (2007). Multilayers for Next Generation X-ray Sources. Report No. UCRL-Conf-230785. Lawrence Livermore National Laboratory, Livermore, CA, USA.

Behrens, C., Schmidt, B., Wesch, S. \& Nicoletti, D. (2010). Proceedings of the First International Particle Accelerator Conference (IPAC'10), Kyoto, Japan, 23-28 May 2010, pp. 912-914. MOPD090.
Feldhaus, J., Grimm, O., Kozlov, O., Plönjes, E., Rossbach, J., Saldin, E., Schneidmiller, E. \& Yurkov, M. (2005). Proceedings of the 27th International Conference on Free Electron Lasers (FEL2005), Stanford, CA, USA, 21-26 August 2005, pp. 183-186. MOPP055

Först, M., Gensch, M., Riedel, R., Tavella, F., Schneidmiller, E. A., Stojanovic, N. \& Yurkov, M. V. (2011). Optical Afterburner for a SASE FEL: First Results from FLASH. Proceedings of the 2nd International Particle Accelerator Conference (IPAC2011), San Sebastián, Spain, 4-9 September 2011. THPC84

Frühling, U., Wieland, M., Gensch, M., Gebert, T., Schütte, B., Krikunova, M., Kalms, R., Budzyn, F., Grimm, O., Rossbach, J. \& Plönjes, E. (2009). Nat. Photon. 3, 523-528.

Geloni, G., Kocharyan, V., Saldin, E., Schneidmiller, E. \& Yurkov, M. (2009). Nucl. Instrum. Methods Phys. Res. A, 605, 409-429.

Gensch, M., Bittner, L., Chesnov, A., Delsim-Hashemi, H., Drescher, M., Faatz, B., Feldhaus, J., Fruehling, U., Geloni, G. A., Gerth, C., Grimm, O., Hahn, U., Hesse, M., Kapitzki, S., Kocharyan, V., Kozlov, O., Matyushevsky, E., Morozov, N., Petrov, D., Ploenjes, E., Roehling, M., Rossbach, J., Saldin, E. L., Schmidt, B., Schmueser, P., Schneidmiller, E. A., Syresin, E., Willner, A. \& Yurkov, M. V. (2008). Infrared Phys. Technol. 51, 423-425.

Golz, T., Pan, R., Zapolnova, E., Schulz, M., Riedel, R., Green, B., Kovalev, S., Gensch, M., Petrovic, J., Rabasovic, M., Krmpot, A., Vladkovic, A. \& Stojanovic, N. (2018). In preparation.

Green, B. et al. (2016). Sci. Rep. 6, 22256.

Grimm, O., Schreiber, S. \& Klose, K. (2006). Proceedings of the 10th European Particle Accelerator Conference (EPAC 2006), Edinburgh, UK, 26-30 June 2006. THPCH150.

Jamison, S. P., Berden, G., Gillespie, W. A. \& MacLeod, A. M. (2008). Proceedings of the 11th European Particle Accelerator Conference (EPAC 2008), Genoa, Italy, 23-27 June 2008. TUPC042.

Jiang, Z. \& Zhang, X. C. (1998). Appl. Phys. Lett. 72, 1945-1947.

Kovalev, S., Green, B., Golz, T., Maehrlein, S., Stojanovic, N., Fisher, A. S., Kampfrath, T. \& Gensch, M. (2017). Struct. Dyn. 4, 024301 .

Nagler, B. et al. (2009). Nat. Phys. 5, 693-696.

Oelze, T., Schütte, B., Müller, M., Müller, J. P., Wieland, M., Frühling, U., Drescher, M., Al-Shemmary, A., Golz, T., Stojanovic, N. \& Krikunova, M. (2017). Sci. Rep. 7, 40736.

Pan, R. (2015). Doctoral dissertation, University of Dundee, UK.

Richter, M., Amusia, M. Y., Bobashev, S. V., Feigl, T., Juranić, P. N., Martins, M., Sorokin, A. A. \& Tiedtke, K. (2009). Phys. Rev. Lett. 102, 163002.

Richter, M., Bobashev, S. V., Sorokin, A. A. \& Tiedtke, K. (2010). J. Phys. B, 43, 194005.

Rönsch-Schulenburg, J., Faatz, B., Honkavaara, K., Kuhlmann, M., Schreiber, S., Treusch, R. \& Vogt, M. (2017). Proceedings of the 8th International Particle Accelerator Conference (IPAC'17), Copenhagen, Denmark, 14-19 May 2017, pp. 2615-2617.

Steffen, B. R. (2007). Thesis, DESY, Hamburg, Germany (No. DESYTHESIS-2007-020).

Stojanovic, N. \& Drescher, M. (2013). J. Phys. B, 46, 192001.

Svetina, C., Mahne, N., Raimondi, L., Perucchi, A., Di Pietro, P., Lupi, S., Schmidt, B. \& Zangrando, M. (2016). J. Synchrotron Rad. 23, 106-110.

Tavella, F., Stojanovic, N., Geloni, G. \& Gensch, M. (2011). Nat. Photon. 5, 162-165.

Tiedtke, K., Feldhaus, J., Hahn, U., Jastrow, U., Nunez, T., Tschentscher, T., Bobashev, S. V., Sorokin, A. A., Hastings, J. B., Möller, S., Cibik, L., Gottwald, A., Hoehl, A., Kroth, U., Krumrey, M., Schöppe, H., Ulm, G. \& Richter, M. (2008). J. Appl. Phys. 103, 094511. 